\title{
LAS POLÍTICAS PARA INCENTIVAR EL RETORNO, LA MOVILIDAD Y LA TRANSFERENCIA DE LA TECNOLOGÍA Y DEL SABER DE LA DIÁSPORA CUALIFICADA. EL CASO DE MARRUECOS*
}

\section{POLICIES TO ENCOURAGE RETURN, MOBILITY AND TRANSFER OF TECHNOLOGY AND KNOWLEDGE OF THE QUALIFIED DIASPORA. THE CASE OF MOROCCO}

Khadija Yahya y María Silvestre Cabrera**

Resumen: En un mundo globalizado, las personas cualificadas son cada vez más solicitadas en todos sitios. Estamos asistiendo a un fenómeno que, según algunos estudiosos, beneficia a los países del norte y perjudica a los del sur. Por ello, algunos de estos últimos países han implementado políticas para incentivar la movilidad y la transferencia de la tecnología y del saber de la diáspora cualificada. Marruecos es uno de los países conscientes de los perjuicios de este fenómeno y ha procedido a la implementación de políticas, a través de la creación de organismos estatales e instituciones, para promover los vínculos con su diáspora y hacer de ella un instrumento de desarrollo del país de origen, así como para construir y consolidar puentes con las redes de la diáspora con el fin de que puedan contribuir al desarrollo de Marruecos.

Palabras clave: diáspora cualificada marroqui; migración cualificada; transferencia del saber; circulación de talentos; Marruecos.

Este trabajo forma parte de una tesis doctoral.

Contacto: <khadijayahya8@gmail.com>,<maria.silvestre@deusto.es>.

*** Universidad de Deusto. 


\begin{abstract}
In a globalized world, qualified professionals are increasingly sought everywhere. We are witnessing a phenomenon, which, according to some experts, benefits Northern countries and penalizes the Southern ones. Therefore, some of the latter countries have implemented policies to encourage mobility and transfer of technology and knowledge of the qualified diaspora. Morocco is a country that has become aware of the damages of this phenomenon and has proceeded through the implementation of policies and the creation of state agencies and institutions in order to promote ties with its diaspora and make it an instrument of development for the country, as well as to build and strengthen the bridges with the diaspora networks to make them contribute to the development of Morocco.
\end{abstract}

Key words: qualified moroccan diaspora; skilled migration; transfer of knowledge; brain circulation; Morocco.

\title{
1. INTRODUCCIÓN
}

El estudio de la movilidad de los recursos cualificados marroquíes en el extranjero es relativamente reducido en la literatura científica debido a que tradicionalmente las migraciones marroquíes se han producido por motivos masivamente económicos y concernían a perfiles poco cualificados (El Asri, 2013). Así, el objeto de este trabajo es arrojar luz sobre la diáspora cualificada marroquí y la promoción - por parte del Estado marroquí- de su retorno, movilidad y la transferencia de tecnología y de saber, la metodología utilizada ha sido el análisis de las políticas implementadas por el gobierno marroquí para vincular su diáspora cualificada inmigrada con el país de origen, siendo la población objeto de estudio la diáspora cualificada perteneciente a la primera generación.

En primer lugar, se presentan algunas de las definiciones de la migración cualificada. En segundo lugar, nos hemos centrado en la conceptualización de la diáspora cualificada, recogiendo conceptos como fuga de cerebros o transnacionalismo, para dar una visión panorámica de la evolución terminológica; también hemos visto la incidencia del fenómeno en los países de destino y de origen, en los primeros centrándonos en la implementación de las políticas migratorias para atraer talento y, en los segundos, analizando la implementación de políticas para incentivar el retorno, intercambio 
o circulación del saber mediante prácticas transnacionales. En tercer lugar, exponemos el estado de la cuestión de la diáspora marroquí cualificada; y, por último, nos hemos centrado en las políticas de Marruecos para incentivar la movilidad y la transferencia de la tecnología y del saber de su diáspora cualificada. En este último apartado, analizamos, además de las políticas públicas, las prácticas transnacionales de las asociaciones de la diáspora cualificada marroquí a favor de la transferencia del saber y la contribución al desarrollo del país de origen, y el papel que juega el gobierno marroquí al respecto.

\section{DEFINICIÓN DE LA MIGRACIÓN CUALIFICADA}

Según Pellegrino (2001), los diagnósticos sobre la migración cualificada suelen adoptar diversas definiciones sobre el tipo de especialistas incluidos en esta denominación, así como sobre la gama de niveles que esta abarca. En la información censal (Martínez, 2005), a las personas cualificadas se les operacionaliza como profesionales, técnicos y afines (PTA); lo que permite identificar los volúmenes y tendencias de la migración cualificada.

Según Khadria (2007), la migración cualificada se refiere al «éxodo de talento y calificación, la parte superior de los profesionales altamente calificados hacia los países desarrollados, que incluye médicos, ingenieros, científicos, profesores, arquitectos, empresarios y otros». En esta línea, González de Aguilar y Valbuena (2011) afirman que se han considerado trabajadores altamente cualificados a los profesionales con titulación universitaria o que ocupen puestos relevantes.

Según la Organización Internacional para las Migraciones $(\mathrm{OIM})^{1}$, la migración cualificada es la «emigración de personas capacitadas o talentosas de su país de origen a otro país, motivada por conflictos o por falta de oportunidades».

1 www.iom.int 


\section{LA MIGRACIÓN CUALIFICADA: ENTRE LA FUGA DE CEREBROS Y LA CIRCULACIÓN DE TALENTOS}

La movilidad cualificada se lleva a cabo en todos los sentidos, pero principalmente desde los países del sur hacia los del norte. Existen varios términos para designar este tipo de movilidad, entre los que cabría señalar "migración de competencias», "desplazamiento de profesionales expertos», "fuga de cerebros», «brain drain», etc. (Elmadmad, 2010). Según Esparragoso (2012), el «brain drain» o la "fuga de cerebros» se refiere a la emigración de personas cualificadas desde países en desarrollo hacia países desarrollados como Estados Unidos y Europa, principalmente.

No se trata de un fenómeno nuevo, pero es a partir del final de la Segunda Guerra Mundial, de la mano del proceso de descolonización de los países africanos, asiáticos y del Caribe en la década de los cincuenta, cuando la preocupación por la migración de las personas cualificadas adquiere relevancia en el ámbito académico y en las discusiones de los organismos internacionales (Pellegrino, 2001).

En la década de los sesenta y de los setenta vieron la luz varios trabajos sobre este fenómeno que se caracterizaron por dos orientaciones: «internacionalistas» $\mathrm{y}$ «nacionalistas». Es de destacar el libro de Walter Adams $(1968)^{2}$, que recoge las ponencias sobre brain drain de Lausana de 1967, reunión que constituyó un evento de importancia y que formalizó la polémica sobre el impacto de la migración cualificada.

El tema de la migración cualificada se introdujo también en la agenda de organismos internacionales como Naciones Unidas, en concreto en la UNCTAD y en la Organización Internacional para las Migraciones, donde esta cuestión formó parte de los debates sobre las relaciones entre las naciones, fundamentalmente en el diálogo Norte-Sur. En este sentido, en la Conferencia de las Naciones Unidas sobre Comercio y Desarrollo celebrada en Santiago de Chile en 1972 se adoptó la denominación «transferencia inversa de tecnología», que optaba por incluir el problema en el marco de tres temas fundamentales: el comercio, la transferencia de recursos y de tecnología y evitar así el contenido emotivo que implicaba el concepto "fuga de cerebros» (Pellegrino, 2001).

\footnotetext{
2 Adams, Walter (1971), El drenaje de talento. Bs. As., Paidós.
} 
A partir de la década de los noventa, debido a los cambios demográficos y al avance del desarrollo tecnológico, el tema volvió a cobrar protagonismo (Parra, 2011). La migración cualificada ha adquirido patrones renovados mediante nuevos flujos desde los países del sur que han ganado fuerza en respuesta a iniciativas que buscan captar mano de obra cualificada para los países del norte. Este proceso se refleja en la ampliación del programa de visas H-1B, por el que se contrata a profesionales altamente cualificados para trabajar temporalmente en Estados Unidos. De hecho, de 1990 a 2003 se ha pasado de 65000 inmigrantes cualificados admitidos a 360498 permisos $\mathrm{H}-1 \mathrm{~B}$ a trabajadores temporales licenciados (Bermúdez, 2010).

En esa época, destaca el predominio de perfiles profesionales de personal altamente cualificado orientado a la ingeniería, las matemáticas, la biotecnología, las tecnologías de la información, la salud y las ciencias. Este nuevo patrón migratorio está muy relacionado con las condiciones surgidas con la globalización, vinculadas a las transformaciones tecnológicas e innovaciones que marcaron una nueva era en el desarrollo científico, y se caracteriza por disciplinas como la robótica, la biotecnología y la informática, enmarcadas en la llamada Economía del Conocimiento, que afectaron a todos los niveles de la economía y la sociedad (Aragonés y Salgado, 2014).

Después del auge de los estudios sobre la «fuga de cerebros», este tema fue perdiendo relevancia en el ámbito académico y en el discurso político. En los últimos años, se ha reavivado la discusión sobre la importancia del conocimiento como elemento básico para el desarrollo, y la reflexión en torno a la consolidación de sectores fuertes en ciencia y tecnología por parte de los gobiernos (Pellegrino, 2001).

Así pues, de una visión negativa o de pérdida, se pasó a otra visión que reivindicaba los aspectos positivos de la movilidad de las personas cualificadas. Desde esta perspectiva, se buscaba transformar el concepto "brain drain» en «brain gain», ya que mediante los retornos transitorios de las migraciones cualificadas circulares o pendulares se contribuye al desarrollo de los países de origen y a la consolidación de sus mercados de trabajo (Pellegrino, 2001). Asistimos entonces a la asunción de nuevas denominaciones tales como «brain gain», «brain circulation», «brain exchange» (Pellegrino, 2001) y «win-win» o «triple-win» (Tung, 2008). 
El transnacionalismo va a ser un nuevo término utilizado para entender el fenómeno de la migración cualificada. Un estudio pionero sobre este concepto es el de Basch, Glick, Schiler y Szanton Blanc (1944), quienes afirman que el transnacionalismo va más allá del nacionalismo ampliado y se refleja en la capacidad de construir redes sociales transnacionales, así como alianzas políticas, económicas e ideológicas, cruzando las fronteras geográficas y culturales de los países tanto de origen como de destino.

Es importante mencionar que el transnacionalismo representa una perspectiva novedosa, pero no un fenómeno nuevo, puesto que se pueden encontrar varios casos de transnacionalismo en la historia de la inmigración, pero lo que es indudable es que las nuevas tecnologías de las telecomunicaciones le han dado un gran impulso a este fenómeno (Portes, 2005).

La perspectiva transnacional va a surgir a finales de la década de los 80 como interpretación alternativa del proceso migratorio. Esta perspectiva no concibe a este como dicotómico-unidireccional o bidireccional; sino que lo entiende como un proceso dinámico de construcción y reconstrucción de redes sociales que estructuran la movilidad espacial y la vida laboral, social, cultural y política, tanto de las personas migrantes como de familiares, amistades y comunidades en los países de origen o destino. Presenta una interpretación más compleja, aunque menos precisa y categórica del proceso migratorio que las teorías de enfoque tradicional (Guarnizo, 2006).

La «circulación de talentos» se produce cuando profesionales así como estudiantes- abandonan sus países de origen de forma transitoria (Esparragoso, 2012). Según este autor, algunos estudios recientes señalan que la globalización ha marcado una nueva tendencia de las migraciones cualificadas en las que el predominio de los flujos temporales de trabajo es predominante. De lo contrario, en países como Estados Unidos se asiste a la prevalencia de los viajes sin retorno.

En 1974, en el marco de la Organización Internacional para las Migraciones (OIM), se puso en marcha el Programa para el Retorno de Personal Cualificado Latinoamericano con el «objetivo de promover y facilitar el retorno ordenado y planificado de personal científico, profesional y técnico latinoamericano que reside en el exterior en general y particularmente en países industrializados». Este programa tuvo tres objetivos fundamentales: facilitar el retorno y la reintegración efectiva de profesionales y personal técnico 
latinoamericano formados en el exterior; incorporar estos recursos cualificados a tareas de desarrollo nacional, subregional y regional a efectos de lograr una transferencia constante de conocimientos y experiencias al personal local con quien trabajan; y satisfacer las necesidades de recursos humanos de alto nivel no disponibles localmente (Pellegrino, 2001).

En la actualidad, las iniciativas tradicionales en materia de la emigración han sido sustituidas o complementadas por otras, cuyo propósito es favorecer los procesos de revinculación y estimular la formación de redes, como manera de transformar las pérdidas en posibles ganancias, tal como se ha señalado anteriormente. Es en el sector académico y científico precisamente donde es factible la cooperación, y es en el que existen posibilidades de intercambio y de transferencias de conocimientos (Pellegrino, 2001).

En definitiva, la situación de la crisis mundial dio paso a un nuevo patrón de acumulación y, a su vez, a nuevos patrones migratorios. En este nuevo patrón es de esperar que las/los trabajadores altamente cualificados no solo prevalezcan, sino que su importancia se refuerce en los mercados laborales internacionales, debido al convencimiento de las potencias de que uno de los mecanismos que pueden favorecer la salida de la crisis pasa por profundizar las condiciones de la economía del conocimiento; para lo cual, necesitan grandes contingentes de este colectivo que hasta ahora era escaso en sus países (Aragonés y Salgado, 2014). Según Parra (2011), se está asistiendo a una competición global por el talento que involucra a actores e intereses diversos; ese interés que se limitaba a países de tradición migratoria como Canadá y Estados Unidos se ha trasladado a Europa y a España, traduciéndose en una serie de medidas y discursos enfocados a facilitar la entrada a las personas migrantes cualificadas.

\section{LA DIÁSPORA CUALIFICADA MARROQUí}

Existen varios términos para designar a la comunidad marroquí residente en el extranjero: los marroquíes del exterior, los marroquíes del mundo, la diáspora marroquí, los marroquíes emigrados, etc., pero sobre todo los marroquíes residentes en el extranjero (les Marocains résidants a l'Étranger: MRE). El perfil de la comunidad marroquí ha cambiado mucho con el tiempo, y es cada 
vez más cualificada. Según datos estadísticos de 2008 (Khachani, 2010), al emigrar, un 10,4\% de la población emigrante tenía un nivel educativo superior; en el caso de España, un 10,2\% contaba con un nivel educativo superior al emprender el proyecto migratorio, y en el año 2005, el nivel superior era el 12,6\%.

En las últimas décadas, se asiste a una diversificación del perfil educativo de la comunidad marroquí instalada en España. Encontramos desde personas sin estudios hasta personas con postgrado (Aparicio et al., 2005). Tal diversificación guarda relación con la zona geográfica de residencia y la edad (Aparicio et al., 2005), así como con las zonas de residencia en el país de origen (Cohen y Berriane, 2011). De hecho, en ciudades como Barcelona predomina el perfil de personas con nivel educativo superior $(27 \%)$ y, por el contrario, en Huelva se observa una cualificación académica particularmente baja. En cuanto a la edad, se observa que cuanto más elevada es la franja de edad, mayor es el porcentaje de personas iletradas (Aparicio et al., 2005). Asimismo, las personas originarias de municipios marroquíes de tamaño menor suelen presentar niveles de estudios inferiores y viceversa (Cohen y Berriane, 2011).

Los recursos cualificados marroquíes emigran sobre todo por el trabajo, ya que las condiciones laborales de las que se benefician en los países de acogida son generalmente mejores que las que les ofrece el país de origen (Elmadmad, 2010). Estos eligen emigrar, pero son sobre todo quienes les emplean quienes los escogen. En la actualidad - a diferencia de lo que ocurría en años anteriores-, las personas marroquíes solicitadas en Europa y en América del Norte son en su mayoría personas con buena formación universitaria y, a menudo, cuentan también con una experiencia laboral confirmada (Elmadmad, 2010).

La proliferación de las agencias de inmigración que operan actualmente en Marruecos —sobre todo canadienses-, así como la especificidad de las condiciones exigidas para la elección (alto nivel educativo, larga experiencia laboral, ser joven, conocimiento de lenguas extranjeras, etc.), son un ejemplo del desarrollo de la migración elegida y la incitación a la fuga de cerebros. Estas agencias ofrecen sus servicios en las grandes ciudades marroquíes como Casablanca y Rabat, además de en páginas web (Elmadmad, 2010).

En ocasiones, la emigración cualificada es incluso la consecuencia de una política gubernamental irreflexiva. De hecho, un ejemplo de 
ello es la operación Salidas Voluntarias (Départs Volontaires) iniciada por el Ministerio de la Función Pública en 2005, que permitió a los altos cuadros de la administración marroquí, así como a grandes expertos, dejar sus funciones antes de la edad de jubilación, al dotarles de una importante indemnización. Algunas de las personas que se beneficiaron de esta operación emigraron a Europa y a América del Norte, sobre todo a Canadá (Elmadmad, 2010).

Las personas que obtuvieron una titulación universitaria en Marruecos o en otros países en los años 60 y 70 se insertaron con facilidad en el mercado laboral marroquí, pero fue a partir de los años ochenta cuando el Estado marroquí - principal generador de puestos de trabajo- disminuyó estas ofertas debido al programa de ajuste estructural realizado en el país, lo que llevó a muchas personas con título a emigrar o a quienes, habiendo proseguido sus estudios en el extranjero, a quedarse en esos países (Khachani, 2010).

La migración de las personas cualificadas marroquíes constituye - en muchos casos- la única solución a los problemas encontrados en el país de origen, y la única alternativa para una vida mejor; al igual que muchas personas desarraigadas, la población migrante marroquí se siente generalmente unida a su país y no lo habría dejado si hubiera encontrado en él las mismas condiciones de vida y de trabajo, así como los mismos salarios que le ofrece su país de residencia (Elmadmad, 2010). No podemos no mencionar otros razones para migrar relacionadas con la búsqueda de una garantía de justicia, libertad e igualdad de trato (Elmadmad, 2010).

El tema de la movilidad de los recursos cualificados marroquíes en el extranjero es relativamente reducido en la literatura científica. En lo que concierne a la literatura prolífica de instituciones internacionales como PNUD, ONU, OMI, etc., consagrada a la movilidad de las diásporas y el papel de estas en el desarrollo de los países del sur, Marruecos parece estar poco cubierto por las tipologías y los modelos tratados (El Asri, 2013). El estudio del fenómeno concerniente a Marruecos tiene carácter reciente, se remonta a los inicios de 2000. Varios motivos explican la escasez así como la aparición reciente de las primeras publicaciones y estudios (El Asri, 2013):

1. Las migraciones marroquíes han sido masivamente económicas, concernían a perfiles poco cualificados y su presencia se ha considerado como sistemáticamente temporal. Esta presencia no entraba en el marco de una reflexión sobre la movili- 
zación de las personas cualificadas, sobre todo la relacionada con las movilizaciones de perfiles altamente cualificados.

2. Los problemas sociales, culturales y económicos, la cuestión de la integración y las discriminaciones encontradas por las poblaciones inmigradas en Europa han focalizado las financiaciones de la investigación sobre estas temáticas, ocultando otras vertientes de la migración y sus efectos sobre los países de origen.

3. Los estudios existentes desde mediados de los años setenta sobre los beneficios de la emigración se han concentrado en una aproximación financiera y cuantitativista, a través de la cuestión de las transferencias financieras como único recurso aportado por la diáspora.

4. Las transformaciones que ha conocido la comunidad marroquí en el extranjero desde hace una veintena de años, como el nivel creciente de cualificación de las personas emigrantes, el éxodo persistente de personal científico y de cuadros altamente cualificados, así como la emergencia progresiva de una élite socioeconómica entre las generaciones resultantes de la migración económica de los años sesenta y setenta han llamado la atención sobre un potencial poco explotado de la diáspora.

Según la base de datos de Docquier, Marfouk y Lowell (2005, en El Asri, 2013), en 1990 había 88.830 activos marroquíes altamente cualificados residentes en los países de la OCDE (12\% de los marroquíes residentes en los países de la OCDE). En el año 2000, este colectivo ha alcanzado las 156.120 personas (14\%), de las cuales las mujeres constituían el $34,4 \%$ en 1990 y el $37,7 \%$ en el 2000 . Además, el nivel educativo de las personas migrantes marroquíes ha conocido una clara mejora: se asiste a una bajada en el número de emigrados «débilmente cualificados» por los emigrados cuyo nivel educativo es medio y alto. Esta tendencia se constata tanto en hombres como en mujeres, aunque los niveles educativos de ellas son algo inferiores.

Según un informe de la OCDE (2012) sobre las diásporas (Bouoiyour, 2013a), la fuga de cerebros es inquietante en el caso de Marruecos. De hecho, la tasa de expatriación hacia los países de la OCDE en 2005-2006 ha sido del 12\%, con lo que Marruecos se sitúa en segundo lugar después de Líbano (12\%). Además, Marruecos ocupa el primer puesto en cuanto a la tasa de emigración de personal médico: en el año 2000, el 28\% del personal médico 
marroquí ejercía en el extranjero. En cuanto a la emigración de personal de enfermería, la tasa era del $20,5 \%$.

Es más, una parte importante de quienes se diploman en las escuelas superiores más prestigiosas de Marruecos emigra cada año (Elmadmad, 2010), y más del 15\% del alumnado marroquí se encuentra en el extranjero; hablamos de unos 50.000 estudiantes (Projet EMEMI, 2009).

En este sentido, como los talentos más solicitados en el mundo entero son los que cuentan con una formación en sectores relacionados con las tecnologías, sobre todo las NTIC, las personas cualificadas marroquíes que interesan más son aquellas que se han formado en tres de las Escuelas más prestigiosas: l'École Mohammedia des Ingénieurs (EMI), l'École Nationale Superieure d'Informatique et d'Analyse de Systémes (ENSIAS) y l'Institut Nationale des Postes et Télécommunications (INPT). Un gran número de egresados y egresadas de estas escuelas emigra cada año para ejercer su talento fuera de Marruecos (Khachani, 2010).

Según datos de la UNESCO (El Asri, 2013), ha habido un incremento de la población estudiantil marroquí inscrita en la enseñanza superior extranjera. Esta cifra ha venido aumentando desde 1999, y conoció su máximo en 2003. En 2009, un $72 \%$ del alumnado marroquí estuvo inscrito en establecimientos franceses, un $10 \%$ en Alemania, un 8,42\% en España, un 3,6\% en Estados Unidos y un 2,27\% en Ucrania. Además, según datos de la OCDE (2009 en Bouoiyour, 2013a), hay 40.700 estudiantes marroquíes en movilidad internacional en los países de la OCDE, situándose décimo en el ranking; si no consideráramos a los países más poblados, como China e India, ni a los países más desarrollados, Marruecos se situaría en segundo lugar después de Malasia (46.700 estudiantes).

Según Bouoiyour (2010), existen al menos cuatro categorías de la diáspora científica y técnica marroquí:

1. Las «estrellas»: se trata de las personas científicas de alto nivel, como por ejemplo profesorado de clases excepcionales, directoras/es de laboratorios de investigación, etc., y que dieron pruebas en el dominio de especialización respectivo.

2. Las personas científicas de alto nivel: generalmente, menores de cincuenta años; comprenden profesorado o profesorado asociado, jóvenes directoras/es de investigación, jefas/es de 
empresas innovadoras, etc. y la duración de su migración es de entre 10 y 16 años.

3. Las/os doctorandas/os, las/os doctoras/es ingenieras/os o las/ os jóvenes profesoras/es encargadas/os de la investigación.

4. Las personas migrantes de segunda y tercera generación.

En el proceso migratorio de las personas cualificadas procedentes de Marruecos ha habido destinos privilegiados que han provocado la concentración de la mayoría de este colectivo; el caso de Francia es significativo en este sentido. De hecho, un 31\% de este colectivo residía en Francia en 1990 (34,75\% en 2000) (base de datos de Doquier Marfouk-Lowell, 2005, en El Asri, 2013). En las últimas décadas, países como Canadá, Estados Unidos y Reino Unido se están convirtiendo en nuevas y atractivas opciones para la diáspora cualificada marroquí.

El perfil de la migración marroquí en Canadá es el de una mujer joven y cualificada. Pueden establecerse cuatro grandes categorías: trabajadoras/es cualificados que tienen un nivel educativo bastante elevado; reagrupación familiar; la población estudiantil $\mathrm{y}$, en menor medida, inversores y emprendedores (Abdelmoula, 2014). A diferencia de Canadá, en Estados Unidos predomina la masculinización de la migración marroquí, aunque también es joven y está cualificada. La mayoría posee título de licenciatura y ha cursado estudios de postgrado (Beveridge et al., 2014).

En el caso del Reino Unido, la política de migración selectiva, que exige una elevada cualificación, —al igual que lo hace la canadiense-ha tenido impacto en el perfil de las personas marroquíes que se dirigen a este país; el perfil actual es el de profesionales altamente cualificados, además de la población estudiantil. Los principales destinos escogidos para estudiar son Francia (donde un 22\% de la comunidad marroquí tiene cualificaciones de nivel superior) e Inglaterra (en Londres el porcentaje alcanza el 31\%) (Cherti, 2014).

Con respecto a la inserción laboral de la diáspora cualificada, en Canadá se observan algunas dificultades respecto a la adecuación entre cualificación e inserción laboral debido sobre todo la falta de experiencia en la sociedad de destino, el problema de la homologación de los títulos académicos y la no posibilidad de formar parte de los Colegios Profesionales, entre otros, lo que da lugar a una sobrecualificación sistemática (Vatz, 2009). 
En Estados Unidos, el porcentaje de la diáspora cualificada marroquí es inferior en los puestos de trabajo directivos y de responsabilidad. Ello se debe, seguramente a la diferencia en el nivel de instrucción (más alto), particularmente en lo que concierne a la obtención de títulos universitarios, en comparación con otros grupos étnicos, excepto para otro países africanos (Beveridge et al., 2014). De hecho, un gran porcentaje se ocupa en la rama «técnicos, comercio, empleado de oficina, etc.» (51\%), mientras el resto se ocupa en las ramas de "dirección y puestos de responsabilidad» $(25 \%)$ y «agricultura, producción y transporte» (24\%), en una proporción casi igual (Censo de los EE.UU. 2007-2011, en Beveridge et al., 2014). A su vez, la diáspora marroquí instalada en Londres se ocupa, primordialmente, en los hoteles y restaurantes $(23,7 \%)$, comercio al por mayor y al por menor $(14,6 \%)$ y en la inmobiliaria $(13,9 \%)$ (Greater Authority 2005 en Cherti, 2014).

Khachani (2010) describe lo que supone la emigración cualificada de Marruecos en clave de pérdida a tres niveles: a) obstaculiza el proceso de desarrollo duradero de las élites; b) se pierde la inversión realizada en su educación y: c) el país se ve obligado a costear a personas expertas extranjeras para el desempeño de empleos cualificados.

Según Elmadmad (2010), la preocupación legítima de Marruecos por el fenómeno de la emigración cualificada no le habilita para impedirla, puesto que Marruecos, como la mayoría de países, ha ratificado gran parte de los principios e instrumentos legales para garantizar los derechos humanos, incluido el de la libertad de movimiento.

Conviene destacar que, desde la llegada al trono del rey Mohamed VI en 1999, se ha producido el retorno de algunas personas de la diáspora cualificada residente en países de Europa y de América del Norte para instalarse definitivamente en Marruecos, incluidas personas pertenecientes a la segunda y tercera generación, o los llamados «ciudadanos europeos migrantes» que, a veces, son objeto de discriminación y exclusión por su origen extra-europeo (Elmadmad, 2010). La justificación del retorno de la diáspora cualificada se debe a que "Marruecos se está expandiendo y las empresas que se instalan en él buscan cuadros formados en Europa y, al mismo tiempo, capaces de comprender la cultura del país»³.

3 Le retour des enfants d'immigrés au Maroc: www. Bladi.net (23/12/2008), en Elmadmad (2010). 


\section{LAS POLÍTICAS DE MARRUECOS PARA INCENTIVAR EL RETORNO, LA MOVILIDAD Y LA TRANSFERENCIA DE LA TECNOLOGÍA Y DEL SABER DE LA DIÁSPORA MARROQUÍ CUALIFICADA}

Marruecos, consciente de la pérdida causada por el establecimiento de parte de su personal cualificado en el extranjero, principalmente en Europa y en América del Norte, busca promover los vínculos con estas personas - así como con el resto de los MREpara que contribuyan al desarrollo de Marruecos, e intenta animar y facilitar su retorno (Elmadmad, 2010).

Para lograr este objetivo, se crearon varias instituciones que tenían como finalidad salvaguardar y desarrollar los vínculos con la diáspora marroquí. Algunas de estas instituciones son (Elmadmad, 2010): El Ministerio Delegado ante el Primer Ministro encargado de la Comunidad Marroquí en el Extranjero; el Consejo de la Comunidad Marroquí en el Extranjero (CCME), la Fundación Hassan II para los Marroquíes Residentes en el Extranjero, la Fundación Mohamed V para la Solidaridad y el Consejo de la Comunidad Marroquí en el Extranjero (CCME).

Existen también otros interlocutores estatales que intervienen en la esfera de la diáspora altamente cualificada, entre otros las Universidades, el Centro Nacional de la Investigación Científica y Técnica (CNRST), el Ministerio de la Enseñanza y de la Investigación Científica, el Ministerio de la Industria, l'Association Marocaine pour la Recherche-Développement R\&D, o la Direction des Investissements Étrangers (DIE) (Bouoiyour, 2008).

El Ministerio Delegado ante el Primer Ministro encargado de la Comunidad Marroquí en el Extranjero se creó en 1990 y, al mismo tiempo, se diseñaron nuevas políticas para fomentar las remesas y las inversiones de las personas marroquíes migrantes. El resultado ha sido una actitud más positiva del gobierno hacia la naturalización, la doble ciudadanía y el derecho al voto de las/os MRE (De Hass, 2007). Uno de los Servicios del Ministerio en la actualidad ataña a la diáspora cualificada; en la página web del Ministerio actualizada se señala cuál es la situación de la diáspora cualificada, se presenta el programa para incentivar su movilidad con el objeto de contribuir al desarrollo de Marruecos, así como la plataforma Maghribcom cuyo objetivo es servir de trampolín para establecer asociaciones «win-win» entre, por una parte, los operadores económicos, las universidades y las instituciones 
de investigación en Marruecos; y, por otra parte, la diáspora cualificada marroquí. Esta plataforma está presente en las redes sociales ${ }^{4}$.

El 8 de enero de 1990, el gobierno marroquí suscribió una convención con el PNUD como uno de los primeros países que se adhirieron al programa TOKTEN. En este marco, Marruecos organizó tres encuentros TOKTEN: en 1993, 1994 y 1996, con la implicación de varios departamentos ministeriales y organismos públicos: los ministerios de Asuntos Extranjeros y la Cooperación, de la Enseñanza Superior, de la Agricultura y de la puesta en valor agrícola, el Ministerio encargado de los Marroquíes Residentes en el Extranjero, el de Transportes, así como instituciones especializadas. Los dominios cubiertos son la ciencia y la tecnología, la energía, la agricultura, la logística y el transporte, y los recursos humanos cualificados científicos y técnicos. Para las tres reuniones, el Ministerio de Asuntos Extranjeros y de Cooperación, a través de la Dirección de la Cooperación Multilateral, sirvió de punto focal y de piloto del programa a nivel nacional (El Asri, 2013).

Ha habido otros encuentros y reuniones, pero el resultado no ha estado al nivel de los medios desplegados, ni a las ambiciones y expectativas generadas (Charef, 2010). Además, los resultados del programa han sido poco conocidos, excepto desde el punto de vista de las recomendaciones, pero ninguna evaluación ha ido más allá. Eso sí, el TOKTEN ha propiciado el marco que ha permitido la creación de una red de personas altamente cualificadas, el reencuentro de las personas galardonadas marroquíes y la publicación de una serie de recomendaciones que, a pesar de todo, han quedado desoídas (El Asri, 2013). Además, se ha creado un anuario de las personas cualificadas marroquíes, que ha permitido el lanzamiento de la asociación Savoir et Développement, así como la constitución de un grupo de investigación muy activo en ingeniería industrial, constituido gracias a los encuentros TOKTEN (Bouoiyour, 2008).

También en 1990, se creó la Fundación Hassan II para los MRE por el Rey Hassan II, y fue presidida por la princesa Lalla Meryem. La Fundación está regida por la ley no 19-89 (13/07/1990), y su objetivo es obrar por mantener los vínculos fundamentales de las/os MRE con su patria, así como ayudarles a superar las dificultades a las que se enfrentan debido a emigración ${ }^{5}$.

\footnotetext{
www.mre.gov.ma

www.fh2mre.ma
} 
En 1999, se creó la Fundación Mohamed V para la Solidaridad, bajo la presidencia efectiva del Rey Mohamed VI, mediante el Decreto del 5 de julio de 1999. La Fundación contribuye, con otros actores sociales, a la lucha contra la pobreza desde varias perspectivas. Entre sus múltiples programas y proyectos cabe señalar la operación de acogida de las personas marroquíes residentes en el extranjero durante el verano (Operación Marhaba).

En 2004, se llevó a cabo una nueva reflexión para incitar y estimular a la diáspora cualificada a contribuir de una manera activa al desarrollo nacional, en el marco de una estrategia global (Charef, 2010). Así, se creó el Programa FINCOME (Fórum Internacional de las Competencias Marroquíes Residentes en el Extranjero) cuyo lanzamiento oficial, en el marco de un evento público, tuvo lugar el 25 de mayo de 2007 (El Asri, 2013). Entre sus objetivos cabe señalar (El Asri, 2013; Bouoiyour, 2008):

- Apoyar la modernización de las empresas marroquíes.

- Apoyar la investigación científica.

- Ayudar a elaborar estrategias sectoriales del desarrollo de Marruecos y a la puesta en práctica y evaluación de proyectos y programas socioeconómicos, científicos y artísticos.

- Promover la atracción de inversiones.

- Ofrecer conocimiento, consejo y asistencia técnica, favorecer la transferencia de la tecnología y del conocimiento y del know-how.

- Contribuir al refuerzo de la cooperación bilateral.

- Acompañar a la diáspora cualificada que desea incorporarse al mercado laboral marroquí.

- Orientar los proyectos potenciales de inversiones de las/os MRE.

FINCOME está dotado de una estructura de funcionamiento encabezada por el Consejo de Dirección y Orientación (CDO) bajo la presidencia del primer ministro, encargado de fijar las grandes orientaciones y acompañar todas las iniciativas y proyectos potenciales de inversión de la diáspora cualificada marroquí. Además, la estructura de FINCOME dispone de la Comisión de Coordinación y Seguimiento (CCS) y la Secretaría Ejecutiva del Consejo y de la Comisión antes citados, además de dos células operacionales de gestión: la primera célula es la célula central de 
gestión, seguimiento y de coordinación, y las siguientes son células de gestión y de seguimiento (El Asri, 2013):

1. La autoridad encargada de la comunidad marroquí residente en el extranjero.

2. La Agencia Nacional de la Promoción del Empleo y de Competencias (ANAPEC).

3. El Centro Nacional de Investigación Científica y Técnica (CNRST).

4. La Célula Central de la Iniciativa Nacional de Desarrollo Humano del Ministerio del Interior (INDH).

5. La Confederación General de las Empresas de Marruecos (CGEM).

6. La Asociación Marroquí para la Investigación-Desarrollo (R\&D Maroc).

El programa FINCOME sigue en activo, está visible en la página web del Centro Nacional de Investigación Científica y Técnica (CNRST), donde constan algunos datos sobre el programa y formularios a rellenar y enviar en caso de interés de formar parte del mismo ${ }^{6}$. Mencionar también que el programa interactúa en algunas redes sociales.

El Consejo de la Comunidad Marroquí en el Extranjero (CCME) fue creado el 21 de diciembre de 2007 como fórmula transitoria con una duración de cuatro años, y sus miembros son cuadros superiores de la diáspora marroquí. Este Consejo se activa para hacer que regresen al país de origen personas marroquíes cualificadas instaladas en el extranjero (Elmadmad, 2010). Según su secretario general, «Marruecos, que está confrontando una falta de recursos humanos altamente cualificados, cuenta entre las filas de su diáspora con un número bastante importante de perfiles que le podrían ayudar a combatir esta carencia» (Elmadmad, 2010). La implementación de este Consejo ha suscitado un caluroso debate en el seno de la comunidad marroquí inmigrada, ya que esta esperaba un órgano que la representara, pero los miembros del consejo han sido nombrados por el rey de Marruecos (Bouoiyour, 2013).

Entre sus principales objetivos cabe destacar la evaluación de las políticas públicas marroquíes y la emisión de opiniones susceptibles de mejorar la eficacia y el rendimiento en beneficio de las comunidades

6 www.cnrst.ma 
marroquíes, la reflexión sobre las modalidades de ampliación de la participación de los MRE en el desarrollo político, económico, social y cultural de Marruecos, y garantizar una función de vigilancia prospectiva sobre las evoluciones de la emigración, los debates que esta suscita tanto en Marruecos como en los países de residencia y en el seno de las instancias internacionales (El Asri, 2013).

Dicho consejo ha organizado varios encuentros con la diáspora cualificada marroquí. Pueden destacarse dos de estos encuentros: el primero tuvo lugar en Canadá el 26 de octubre de 2009 (Elmadmad, 2010), y el segundo encuentro fue sobre las personas cualificadas marroquíes, y se celebró en Barcelona el 24 de abril de 2010; se trató de una iniciativa organizada por el CCME en colaboración con la Federación de las Entidades Culturales Catalanes de Origen Marroquí (FECCOM) ${ }^{7}$.

Además, merece la pena mencionar que se han creado en el seno del CCME algunos grupos de trabajo, como los siguientes (El Asri, 2013):

1. "Competencias científicas y técnicas en el extranjero para el desarrollo solidario» (CSTEDS): Este grupo de trabajo está completamente dedicado a la cuestión de los recursos cualificados marroquíes en el extranjero. Ha adoptado un enfoque participativo basado esencialmente en las consultas de las personas cualificadas por país, por temática, y las consultas ante las instituciones encargadas de la movilización de este colectivo.

2. «Administración, derechos de usos y políticas públicas»: Este grupo ha trabajado sobre la cuestión del mercado de trabajo, del empleo y de la migración, y ha organizado reuniones de trabajo con las instituciones marroquíes encargadas de la cuestión de la migración laboral.

3. «Perspectiva de género y nuevas generaciones»: Este grupo ha organizado durante la segunda edición de «Marroquíes de aquí y de allá» un taller sobre las mujeres cualificadas marroquíes residentes en el extranjero. Además, vela por incluir en cada una de las ediciones de «Marroquíes de aquí y de allá» intervenciones y debates sobre las condiciones de trabajo de las mujeres marroquíes migrantes. En uno de

$7 \quad$ www.ccme.org.ma 
estos encuentros, se afirmó que las mujeres parecen estar más unidas que los hombres a su país de origen y estar más dispuestas a contribuir a su desarrollo (Elmadmad, 2010).

De momento, las atribuciones de CCME no están bien definidas, pero el hecho de que esté compuesto por personas altamente cualificadas da una idea de la nueva visión estratégica ante la problemática migratoria que parecen tener las autoridades marroquíes (Bouoiyour, 2008; Bouoiyour, 2013).

El siguiente cuadro presenta los distintos dispositivos creados para la vinculación de la diáspora marroquí cualificada con su país de origen:

Cuadro 1

PRINCIPALES DISPOSITIVOS CREADOS PARA VINCULAR LA DIÁSPORA CUALIFICADA CON EL PAÍS DE ORIGEN

\begin{tabular}{|l|c|}
\hline \multicolumn{1}{|c|}{ Tipo de dispositivo } & Año de creación \\
\hline Ministerio Encargado de las/os Marroquíes Residentes en el Extranjero & 1990 \\
\hline Programa TOKTEN & 1990 \\
\hline Fundación Hassan II para las/os MRE & 1990 \\
\hline Fundación Mohamed V para la Solidaridad & 1999 \\
\hline Programa FINCOME & 2007 \\
\hline Consejo de la Comunidad Marroquí en el Extranjero (CCME) & 2007 \\
\hline
\end{tabular}

Fuente: elaboración propia.

Además de los programas anteriormente mencionados, el Ministerio encargado de la Comunidad marroquí en el extranjero organizó una universidad de personas cualificadas marroquíes residentes en Alemania del 20 al 22 de noviembre de 2009 en la ciudad marroquí de Fez. El objetivo de esta universidad fue identificar y movilizar a las diferentes personas cualificadas y expertas de la diáspora en Alemania, y hacerlas trabajar con personas auxiliares en Marruecos en diferentes dominios: investigación, desarrollo 
duradero e innovación, sector medical y sanitario, ecología y medio ambiente, sector automovilístico y aeronáutico, desarrollo económico y social, artes y cultura (Elmadmad, 2010).

Respecto a la recuperación física de la diáspora cualificada, cabe destacar que aunque en el discurso oficial no es altamente prioritaria la cuestión del retorno a Marruecos de nacionales instalados en el extranjero, es evidente que algunas políticas puestas en marcha, sobre todo desde 2002 y 2003, contribuyen en cierta manera a crear las condiciones propicias para el retorno de las personas cualificadas marroquíes, aunque ello no pueda ser definido como una completa "política de retorno» de los MRE. Podemos mencionar, por ejemplo (Lahlou, 2006):

1. La Iniciativa Nacional de Desarrollo Humano (INDH), iniciada a partir de 2005, cuyo objetivo es reducir la prevalencia de la pobreza en el medio rural y en los barrios periféricos de las grandes ciudades.

2. Los programas de desintegración de los hábitats insalubres;

3. La puesta en marcha de programas agrícolas, relacionadas con las culturas fruteras y oliveras, por ejemplo, que incitan tanto a la inversión local como a las inversiones extranjeras, de las cuales las/os marroquíes instalados en el extranjero.

4. La adaptación, desde 2003, de un marco de inversión incitativo a la promoción de la pymes, con numerosas facilidades respecto a las/os inversores nacionales, así como a las/os extranjeros y a las/os MRE.

5. La puesta en marcha desde finales de 1990 del «Fonds Hassan II» (Fondos Hassan II) de desarrollo económico y social.

Podría afirmarse que lo que Marruecos estaría esperando es una transferencia de medios financieros, de cualificaciones adquiridas y del conocimiento acumulado en el extranjero de los MRE (Lahlou, 2006). Aunque aún no conste un enfoque oficial global, planificado y voluntario respecto al retorno, un documento firmado por el gobierno marroquí en el marco del encuentro gubernamental sobre las migraciones (julio de 2006) se refiere a la necesidad de facilitar el retorno de los «cerebros», de poner en marcha políticas incitativas al retorno de la población estudiantil y la necesidad de definir políticas, y, al mismo tiempo, garantizar la reinserción de las/os emigrantes retornados. Sin embargo, tales objetivos/compromisos 
no se han traducido en la realidad (Lahlou, 2006). De hecho, en la actual Estrategia del Ministerio Encargado de los Marroquíes Residentes en el Extranjero no figura la cuestión del retorno de la diáspora cualificada entre los objetivos principales de la Estrategia ${ }^{8}$.

Por otra parte, la sociedad civil, a su vez, incita a veces el retorno de las personas cualificadas y contribuye a la lucha contra la fuga de cerebros. Por ejemplo, el Fórum Horizons-Maroc, iniciado por l'Association des Marocains des Grandes Écoles (AMGE), actúa para incitar al retorno a Marruecos a jóvenes diplomados residentes en Francia. Uno de los responsables de la organización de este fórum ha señalado que «el fenómeno gana importancia, y cada vez más jóvenes cualificados están dispuestos a volver a Marruecos, a condición de que se les ofrezca un puesto que se corresponda con sus expectativas» (Elmadmad, 2010). Esta disposición por parte de este colectivo la confirma Bouoiyour (2008), quien considera que cada vez más el personal investigador de origen marroquí que vive en otros países expresa su deseo de participar en la investigación nacional.

Por otra parte, debemos destacar la contribución de la diáspora cualificada en los cambios que ha conocido Marruecos en los últimos años. Por ejemplo, desde principios del 2000, ha influido en algunas reformas jurídicas y sociales del país, como la reforma del Código de la Familia «Mudawana», la revisión del Código de la Nacionalidad, la asociación de las mujeres marroquíes residentes en el extranjero a la vida pública al atribuirles puestos de responsabilidad, etc. Un ejemplo de esto último es el del actual consejero del rey de Marruecos y anterior ministro de Turismo, Yassir Znagui, que ha sido educado en Francia y ha trabajado en Londres antes de retornar a Marruecos (Elmadmad, 2010).

Es de señalar también que las personas cualificadas marroquíes inmigradas se organizan en asociaciones (Bouoiyour, 2008). Se trata de prácticas transnacionales "desde abajo" (Portes, 2005), que conectan a las/os migrantes desde el exterior con su país de origen, al tiempo que se convierten en agentes estructuralmente indispensables para - aunque a menudo rechazados por- la sociedad receptora (Guarnizo, 2009). Generalmente, las prácticas transnacionales de estas asociaciones se inscriben bien en el marco de la estrategia nacional de retorno de la diáspora cualificada marroquí inmigrada y la voluntad

$8 \quad$ www.mre.gov.ma 
de esta de participar activamente en el desarrollo de Marruecos; o bien se trata de asociaciones de la clásica ayuda mutua o de transferencia del conocimiento (Charef, 2010). A este respecto, se puede hablar globalmente de dos tipos de proyectos sostenidos (Bouoiyour, 2008):

1. Los que suponen un alianza entre el personal científico marroquí del extranjero con el que reside en Marruecos - generalmente universitarios- Se trata de proyectos académicos estándares de tipo "acciones integradas» sostenidos por el gobierno marroquí y los gobiernos extranjeros, en los que las asociaciones diaspóricas juegan un rol principal. Existen otras acciones que conciernen proyectos regionales; por ejemplo, una región francesa y una marroquí. Las asociaciones juegan el rol de facilitador, también intentan reorientar los proyectos y disminuir la brecha que pudiera existir entre los responsables marroquíes y los extranjeros en cuanto a la comprensión de las implicaciones de algunos proyectos.

2. El segundo tipo de proyectos se ocupa de ayudar a algunos organismos gubernamentales y a las empresas marroquíes - sobre todo las pymes-. Respecto a estas, el objetivo es enseñarles a innovar de una manera rentable, así como ayudarles a encontrar asociados para este fin, construyendo, así, redes de personas cualificadas entre los socios de diferentes sectores y entre las pymes y las grandes empresas como, por ejemplo, Omnium Nord-African (ONA), o las oficinas del Estado tales como l'Office Chérifien des Phosphates (OCP), l'Office Nacional d'Électricité, etc. Otro polo de interés de estas asociaciones es la colaboración con las instituciones nacionales responsables de la investigación científica. La idea es crear grupos de trabajo pluridisciplinares y multisectoriales, asociando responsables de asociaciones científicas, de empresas, de institutos de investigación y de universidades, para reflexionar en común sobre la implementación de una verdadera política de las ciencias y la tecnología.

Citamos, a continuación, algunas de las asociaciones más dinámicas (Bouoiyour, 2008):

- Savoir et Développement: creada en el año 1999 en la Universidad de Pau y de los Países del Adour. Desde su lanzamiento, reagrupa a más de doscientos expertos a través 
del mundo, y su objetivo es la transferencia de la tecnología hacia Marruecos.

- Regroupement des Biologistes Marocains au Canadá (RMBC): su objetivo es reagrupar a todas/os aquellas/os que obran en el dominio de las ciencias biológicas y las disciplinas anexas, favoreciendo los intercambios entre Marruecos y Canadá.

- Caravane-Marocains des Grandes Écoles.

- BIOMATEC-Association Marocaine de Biologie.

- Maroc-Entreprendre.

- Réseau International des Talents Marocains.

- Migration et Développement.

Generalmente, el objetivo de estos movimientos asociativos es promover los intereses profesionales de sus miembros, pero también su socialización (Khachani, 2010). Sin embargo, las iniciativas de estas asociaciones - por muy importantes que seanquedan dispersas y no han sido lo suficientemente apoyadas por las autoridades marroquíes; además, carecen de coordinación y de información entre sí, lo que hace que sea difícil seguir de cerca sus actividades (Bouoiyour, 2008).

En la actualidad, en la página web del Ministerio encargado de las/os MRE está operativo un servicio destinado al asociacionismo marroquí en el exterior. Se busca poner en práctica una aproximación participativa para promover los interés de las/os MRE, para ello el Ministerio ha desarrollado un ambicioso programa que pretende facilitar la integración de estos en los países de acogida ${ }^{9}$, al promover el asociacionismo de la diáspora cualificada con el fin de contribuir al desarrollo del país de origen.

En resumen, la política marroquí de reintegración de la diáspora cualificada es balbuceante; a pesar de la toma de conciencia de la migración como medio para financiar la economía y disminuir la presión sobre el empleo, lo relativo a la transferencia de la tecnología a través de la diáspora no ha sido suficientemente asimilado y tenido en cuenta (Bouoiyour, 2013). Es posible afirmar que quizás Marruecos no esté lo suficientemente maduro y preparado para atraer a su diáspora, una muestra de ello es la multiplicación de organismos estatales encargados de la emigración que no facilitan la labor a quienes integran la diáspora (Bouoiyour, 2013).

$9 \quad$ www.mre.gov.ma 
Según Bouoiyour (2013), para solucionar esta situación, haría falta lo siguiente: en primer lugar, poner en marcha una política integrada con visión y estrategia de futuro que prevenga la migración cualificada; en segundo lugar, no decepcionar a las diáspora con promesas incumplidas o recursos fallidos (como lo fueron el programa TOKTEN o el FINCOME) y; en tercer lugar, reconsiderar la forma en la que se gestiona la política migratoria en Marruecos.

\section{CONCLUSIONES}

Podemos afirmar que si bien hasta hace poco los flujos migratorios marroquíes respondían fundamentalmente a cuestiones económicas, políticas y de reagrupamiento, en los últimos años ha habido una significativa presencia de emigraciones cualificadas que tienen serias dificultades de inserción laboral en Marruecos motivadas tanto por las dificultades propias de su tejido productivo, como por la complejidad institucional que busca su retorno.

Hemos constatado que a pesar de que la diáspora cualificada marroquí ha sido poco estudiada — debido al predominio de flujos de la migración económica-, su presencia en algunos países de la OCDE es notable. Esta diáspora engloba tanto a personas profesionales como a estudiantes, cuyo número está en aumento. La emigración cualificada marroquí supone una importante pérdida para Marruecos, puesto que no solo invierte en la formación académica de personas que luego emigran, sino que también se ve obligado a contratar a personas expertas extranjeras a un coste elevado para cubrir determinados puestos de trabajo.

Consciente de esta problemática, el Estado marroquí ha buscado promover a través de la implementación de algunas políticas, el retorno, el intercambio o la circulación de la tecnología y del saber de su diáspora cualificada. Para ello, ha creado algunos dispositivos como el CCME, FINCOME o TOKTEN, entre otros. Sin embargo, la política de integración de la diáspora no está dando los frutos esperados, debido, fundamentalmente, a su propia diversidad y complejidad, lo que dificulta la labor a la propia diáspora cualificada. La literatura analizada establece que sería necesaria una visión integrada para el abordaje de esta problemática por parte del Estado marroquí. A muchas personas expertas marroquíes les sorprende 
la ausencia de una verdadera política migratoria marroquí, sobre todo porque el número de estudiantes marroquíes aumenta, y la proporción de los que no retornan crece y, sin embargo, quienes tienen la responsabiliad parecen ignorar este problema, como lo muestra la falta de iniciativas y estudios que calculen el coste de esta nueva movilidad internacional. Se puede hablar, incluso, de una falta de visión clara por parte de las autoridades marroquíes sobre la manera de tratar esta nueva forma de movilidad (Bouoiyour, 2008).

Como afirma Pellegrino (2001), es difícil modificar el volumen y tendencias del fenómeno si no existe una voluntad explícita de los agentes implicados. Por ejemplo, los países de origen de las personas emigrantes cualificadas deberían valorar el ensayo de políticas que busquen la retención o retorno de sus talentos, si valoran que son estratégicos para su desarrollo. Por su parte, los países receptores, es probable que regulen cada vez más el volumen y las características del tipo de personas trabajadoras; de hecho, la implementación de políticas que incentivan la entrada ya están en práctica y responden a la proyección de futura demanda insatisfecha en determinadas áreas del conocimiento.

\section{BIBLIOGRAFÍA}

Abdelmoula, Y. (2014): "Les marocains du Canada", en M. Berriane (dir.), Marocain de l'Extérieur 2013, Rabat: Fondation Hassan II pour les Marocains Résidant à l'Étranger.

Aparicio R., Van Ham, C., Fernández, M. y Tornos, A. (2005): Marroquíes en España, Madrid. Universidad Pontificia Comillas, Migraciones $\mathrm{n}^{\circ} 6$.

Aragonés, A. M. y Salgado, U. (2014): “¿Competencia internacional por la migración altamente calificada?”. Comercio Exterior, 64 (2), pp. 18-26.

Basch, L., Glick Schiller, N. y Szanton Blanc, C. (1994): Nations unbound. Transnational projects, postcolonial predicaments and deterritorialized nation-states. Pensylvania, Gordon and Breach Science Publishers.

Bermúdez Rico, R. E. (2010): "Migración calificada e integración en las sociedades de destino". Sociedad y Economía, 19, pp. 135-150.

Beveridge, A., Weber, S. y Beveridge, S. (2014): "Les marocains des ÉtatsUnis", en M. Berriane (dir.), Marocain de l'Extérieur 2013. Rabat, Fondation Hassan II pour les Marocains Résidant à l'Étranger.

Bouoryour, J. (2008): "Diaspora et développement: Quelles interactions dans le cas marocain?". Migration et Société, 120, CNRS Editions. 
Bouoiyour, J. (2010): “La diaspora scientifique marocaine comme ressource d'expertise et d'enseignement". Management Systems International (MSI), 66 pp.

Bouoryour, J. (2013): "Les transferts des fonds des migrantes marocains: Leviers de croissance et du développement". MPRA Paper, 50537.

Bouolyour, J. (2013a): Stratégie National de l'émigration à l'horizon 2030. Analyse du contexte international de la migration marocaine. Rapport pour le Ministère, IRES et CCME.

Cohen, A. y Berriane, M. (2011): De Marruecos a Andalucía: Migración y espacio social. Granada, Universidad de Granada.

Charef, M. (2010): “Migration des compétences". Rabat, Les Cahiers du Plan, n 29.

Cherti, M. (2014): "Les marocains du Royaume Unis", en M. Berriane (dir.). Marocain de l'Extérieur 2013. Rabat, Fondation Hassan II pour les Marocains Résidant à l'Étranger.

De Hass (2007): “Morocco's migration experience : a transitional perspective”. International Migration, 45 (4), pp. 39-70.

El AsRI, F. (2013): Migrations marocaines. Les compétences marocaines de l'étranger: 25 ans de politiques de mobilisation. Rabat, CCME.

Elmadmad, K. (2010): "La Migration qualifiée au Maroc. Une étude sociojuridique”. Florencia, European University Institute.

Esparragoso Vázouez, R. (2012): "Perspectivas y retos de la migración cualificada latinoamericana. España como país receptor (1999-2009)”. Temas Americanistas, 28, pp. 23-41.

González de Aguilar, C. y Valbuena Reyero, M. R. (2011): "Inmigración altamente cualificada: Mercado y Regulación. Una revisión del caso español”. Anales de Derecho, 29, pp. 81-114.

GuARnizo, L. E. (2006): “Migración, globalización y sociedad: teorías y tendencias del siglo XX”, en G. Ardila (ed.). Migraciones, Transnacionalismo y Desplazamiento. Bogotá, CES, pp. 65-112.

KHACHANI, M. (2010): La migration des personnes hautement qualifiées. Le cas du Maroc. Florencia, European University Institute.

KHACHANI, B. (2007): "India: migración calificada a los países desarrollados, migración laboral al Golfo”. Migración y Desarrollo: perspectivas desde el sur, pp. 87-124.

Lahlou, M. (2006): "Migration de retour au Maroc : une approche socioéconomique et institutionnelle”. MIREM Project, Migration de retour au Maghreb.

Martínez Pizarro, J. (2005): Globalizados, pero restringidos: una visión latinoamericana del mercado global de recurso humanos calificados. Santiago de Chile, United Nations Publications, Vol. 56.

Parra Cartagena, M. C. (2011): "La migración cualificada en España”. Fundación Encuentro, Informe España 2011.

Pellegrino, A. (2001): "Éxodo, movilidad, circulación: nuevas modalidades de la migración calificada”. Notas de Población, 73, pp. 129-162. 
Portes, A. (2005): “Convergencias teóricas y evidencias empíricas en el estudio del transnacionalismo de los inmigrantes". Migración y Desarrollo, 4, pp. 2-19.

Chamkhi, A., Dubois, T. y Soubiran, T. (2009): "Enquête sur la Mobilité Etudiante Marocaine Internationale". Rabat, Projet EMEMI.

Tung, R. (2008): "Brain circulation, diaspora, and international competitiveness”. European Management Journal, 26 (5), pp. 298-304.

Vatz Laaroussi, M. (2009): "Les femmes marocaines immigrantes au Québec: entre désillusion et changement". Rabat, CCME. 
